\section{(6) OPEN ACCESS}

\title{
Carbonic anhydrase IV inhibits colon cancer development by inhibiting the Wnt signalling pathway through targeting the WTAP-WT1-TBL1 axis
}

\author{
Jingwan Zhang, ${ }^{1}$ Ho Tsoi, ${ }^{1}$ Xiaoxing Li, ${ }^{1}$ Hua Wang, ${ }^{2}$ Jing Gao, ${ }^{1,3}$ Kunning Wang, ${ }^{1}$ \\ Minnie YY Go, ${ }^{1}$ Siew C Ng, ${ }^{1}$ Francis KL Chan, ${ }^{1}$ Joseph JY Sung, ${ }^{1}$ Jun $Y u^{1}$
}

- Additional material is

published online only. To view please visit the journal online (http://dx.doi.org/10.1136/ gutjnl-2014-308614).

${ }^{1}$ Institute of Digestive Disease and Department of Medicine and Therapeutics, State Key Laboratory of Digestive Disease, Li Ka Shing Institute of Health Sciences, CUHK Shenzhen Research Institute, The Chinese University of Hong Kong, Hong Kong, Hong Kong ${ }^{2} S$ chool of Biomedical Science, The Chinese University of Hong Kong, Hong Kong, Hong Kong ${ }^{3}$ Department of Gastrointestinal Oncology, Key Laboratory of Carcinogenesis and Translational Research (Ministry of Education), Beijing University Cancer Hospital \& Institute, Beijing, China

\section{Correspondence to} Dr Jun Yu, Institute of Digestive Disease and Department of Medicine and Therapeutics, Prince of Wales Hospital, The Chinese University of Hong Kong, Shatin, NT, Hong Kong; junyu@cuhk.edu.hk

Received 11 October 2014 Revised 19 April 2015 Accepted 21 April 2015 Published Online First 12 June 2015

\section{ABSTRACT}

Objective We found that carbonic anhydrase IV (CA4), a member of the carbonic anhydrases, is silenced in colorectal cancer (CRC). We analysed its epigenetic inactivation, biological effects and prognostic significance in CRC.

Design The biological functions of CA4 were determined by in vitro and in vivo tumorigenicity assays. The CA4 co-operator was identified by immunoprecipitation and mass spectrometry. CA4 downstream effectors and signalling pathways were elucidated by promoter luciferase assay, electrophoretic mobility shift assay and chromatin immunoprecipitation. The clinical impact of CA4 was assessed in 115 patients with CRC

Results CA4 was silenced in all nine CRC cell lines and $92.6 \%$ of CRC tumours. The promoter hypermethylation contributed to the inactivation of CA4, and it was detected in $75.7 \%$ of the patients with CRC. After a median follow-up of 49.3 months, multivariate analysis showed that the patients with CA4 hypermethylation had a recurrence of Stage II/III CRC. The re-expression of CA4 inhibited cell proliferation, induced apoptosis and cell cycle arrest in the G1 phase. CA4 inhibited the activity of the Wnt signalling pathway and mediated the degradation of $\beta$-catenin. CA4 interacted with Wilms tumour 1-associating protein (WTAP) and induced WTAP protein degradation through polyubiquitination.

Moreover, CA4 promoted the transcriptional activity of Wilms' tumour 1 (WT1), an antagonist of the Wnt pathway, which resulted in the induction of transducin $\beta$-like protein 1 (TBL 1) and the degradation of $\beta$-catenin. Conclusions CA4 is a novel tumour suppressor in CRC through the inhibition of the Wnt signalling pathway by targeting the WTAP-WT1-TBL1 axis. CA4 methylation may serve as an independent biomarker for the recurrence of $C R C$.

\section{INTRODUCTION}

Colorectal cancer (CRC) is a malignant disease caused by a variety of factors involving the successive accumulation of genetic and epigenetic alterations. ${ }^{1}$ CRC is still the second most common cancer worldwide. $^{2} 3$ One million new CRC cases are reported annually; the disease-specific mortality rate is nearly $33 \% .^{2}$ Surgical resection is the mainstay of treatment for patients with CRC, but tumour

\section{Significance of this study}

What is already known on this subject?

- Transcriptional inactivation of tumour-suppressor genes through promoter hypermethylation plays an important role in the development of colon cancer.

- Carbonic anhydrase IV (CA4), located on chromosome 17q22, is a member of the family of zinc-containing metalloenzymes that catalyse the reversible hydration of carbon dioxide and the dehydration of carbonic acid.

- CA4 is expressed in normal colon tissue.

What are the new findings?

- Promoter hypermethylation contributes to the silencing of CA4 in colon cancer call lines and tumour tissues.

- CA4 is a tumour suppressor in colon cancer.

- The antitumorigenic function of CA4 is modulated by suppressing the activity of the Wnt signalling pathway through targeting the Wilms' tumour 1-associating protein (WTAP)WT1-TBL1 axis: CA4 promotes the degradation of WTAP and it enhances the WT1-binding activity to induce the expression of transducin $\beta$-like protein 1 (TBL1), and this results in the degradation of $\beta$-catenin.

- CA4 methylation serves as a biomarker for predicting the recurrence of colon cancer.

How might it impact on clinical practice in the foreseeable future?

- Detection of methylated CA4 may serve as an independent biomarker for the recurrence of cancer in patients with colon cancer.
To cite: Zhang J, Tsoi $\mathrm{H}$ Li $X$, et al. Gut 2016;65:1482-1493. recurrence is not uncommon. In a large cohort study, the median survival following CRC recurrence was 13.3 months. ${ }^{3}$ The mechanisms leading to CRC development, progression and recurrence are complex. The downregulation of the tumoursuppressor genes caused by the hypermethylation of C-phosphate-G (CpG) islands in their promoter region is one of the crucial mechanisms in CRC 
development and progression. ${ }^{1}$ DNA methylation has been reported to be a biomarker of cancer recurrence. ${ }^{4-6}$ Thus, the identification of novel tumour-suppressive genes may uncover tumour-suppressive pathways in colorectal carcinogenesis. In addition, these discoveries may identify potential biomarkers for tumour diagnosis and predictors of postoperative recurrence in patients with CRC. ${ }^{7}$

We have recently identified a novel preferentially methylated gene, carbonic anhydrase IV (CA4), located on chromosome $17 \mathrm{q} 22,{ }^{8}$ which is preferentially methylated in human colon cancer through promoter methylation array (see online supplementary table S1). CA4 belongs to a large family of zinccontaining metalloenzymes that catalyse the reversible hydration of carbon dioxide and the dehydration of carbonic acid ${ }^{9}$ and it is reported to be expressed in normal colon tissues. ${ }^{10}{ }^{11}$ The downregulation of CA4's homologues, either CAI or CAII, has been shown to correlate with colorectal carcinogenesis. ${ }^{12}$ However, the role of CA4 in CRC recurrence is still unclear. In this study, we identified that the frequent loss of CA4 expression was due to promoter hypermethylation of the CA4 gene in CRC. Further functional studies revealed that the ectopic expression of CA4 results in a significant suppression of CRC growth by inducing apoptosis and G1 cell cycle arrest. The tumour-suppressive effect of CA4 was found to be associated with the inhibition of the $\mathrm{Wnt} / \beta$-catenin signalling pathway by: (1) the inhibition of the active form of $\beta$-catenin; (2) the direct interaction with Wilms' tumour 1-associating protein (WTAP) and the induction of WTAP degradation via polyubiquitination; (3) the enhancement of Wilms' tumour 1 (WT1)-binding activity, an antagonist of the Wnt pathway; and (4) the induction of the protein expression of transducin $\beta$-like protein 1 (TBL1), a downstream effector of WT1, which in turn contributes to $\beta$-catenin degradation. ${ }^{13}{ }^{14}$ Moreover, CA4 promoter hypermethylation was significantly associated with CRC recurrence. Thus, our results reveal the mechanism and the clinical application of CA4, a novel tumour-suppressive gene, in CRC.

\section{MATERIAL AND METHODS}

CRC cell lines and clinical samples

Colon cancer cell lines (CaCO2, DLD-1, HCT116, HT-29, LOVO, LS180, SW480, SW620 and SW1116) were obtained from the American Type Culture Collection (ATCC, Manassas, Virginia, USA). The colon normal epithelial cell line NCM460 was obtained from In Cell (San Antonio, Texas, USA). Roswell Park Memorial Institute (RPMI) 1640 medium (Gibco BRL, Rockville, Maryland, USA) was used except HCT116 in McCoy's 5A medium (Gibco BRL).

Surgically excised CRC tissues and surrounding non-tumour colon tissues were obtained from 115 patients with CRC at the Beijing University Cancer Hospital. The patient demographics and clinicopathological features are shown in online supplementary table S2. Each tumour was staged in accordance with the primary tumor $(\mathrm{T})$ nearby lymph node $(\mathrm{N})$ metastasis $(\mathrm{M})$ (TNM) staging system. Patients were regularly followed up, and the median follow-up duration since the time of diagnosis was 49.3 months (range 12.4-85.3 months).

\section{Chromatin immunoprecipitation assay}

The chromatin immunoprecipitation (ChIP) experiment was performed, as described previously. ${ }^{15}$ Briefly, stable CA4-expressing HCT116 cells and control HCT116 cells were cross-linked with formaldehyde and then collected for nuclear protein extraction. Chromatin from the extracted cross-linked nuclei was sheared by sonication and precipitated with antibody to WT1 (Abcam); the immunoprecipitated protein-DNA complex was then captured with protein-G magnetic beads. An equal number of cells $\left(1 \times 10^{7}\right)$ was used for each immunoprecipitation. The same amount of non-specific IgG was used as control. After the reversal of the cross-link and digestion of proteins with proteinase K, the immunoprecipitated DNA was isolated by the phenol/chloroform/isoamyl alcohol method. The presence of TBL1 DNA in the immunoprecipitant was examined by PCR by using the primers, as follows: forward, $5^{\prime}$ - thymine, guanine, adenine (TGA) CTG AGC AAG GCG AAA GGA G-3' and reverse, $5^{\prime}$-GTG CAA TAG AAA ACC CGA ACG AGC-3'.

\section{Electrophoretic mobility shift assay}

Electrophoretic mobility shift assay was performed, as reported previously. ${ }^{16}$ Briefly, HCT116 and SW1116 $(4 \times 106$ cells/each $)$ were seeded on $10 \mathrm{~cm}$ dishes and transfected either with $5 \mathrm{~g}$ of pcDNA 3.1 or pcDNA3.1-CA4 by Lipofectamine 2000 . Forty-eight hours post-transfection, the cells were treated with $2 \mu \mathrm{M}$ of MG132 or dimethyl sulfoxide (DMSO) for $6 \mathrm{~h}$ and then fixed, harvested and subjected to sonication in lysis buffer for making the major DNA fragment in 200-300 bp. Anti-WT1 antibody $(1: 100)$ was added to the resulting reaction mixture. The WT1/DNA complex was eluted and analysed by $4.5 \%$ of native polyacrylamide gel. The DNA was stained by SYBR green (Molecular Probe), and the complex in the gel was visualised by UV illumination.

\section{Statistical analysis}

The results were expressed as mean \pm SD. The Mann-Whitney U test was performed to compare the difference of the CA4 protein expression between the tumour and the adjacent nontumour tissues. The difference in the tumour growth rate between the two groups of nude mice was determined by repeated-measures analysis of variance. The $\chi^{2}$ test was used for comparison of patient characteristics and distributions of methylation and covariates by vital status. The patients' ages (at entry to follow-up) were compared by using the $t$ test. Crude risk ratios (RRs) of recurrence associated with CA4 methylation and other predictor variables were estimated by using a univariate Cox proportional hazards regression model. A multivariate Cox model was constructed to estimate the adjusted RR for CA4 methylation. The overall recurrence rate in relation to methylation status was evaluated by the recurrence curve and the log-rank test. Analysis of overall recurrence was limited to a 5 -year period to avoid the probability of the recurrence not being related to CRC. A p value $<0.05$ was regarded as statistically significant (see online supplementary materials and methods).

\section{RESULTS}

\section{CA4 is downregulated in primary colorectal tumours}

We first examined the CA4 expression in normal human tissues and found that CA4 was expressed in most of the normal human tissues, including those in the colon (see online supplementary figure S1). However, CA4 mRNA expression was significantly downregulated in $92.6 \%(25 / 27)$ of the CRC tumour tissues compared with their adjacent non-tumour tissues $(\mathrm{p}<0.0001$; figure $1 \mathrm{~A})$. Consistently, the CA4 protein level was reduced in the CRC tumour tissues compared with their adjacent non-tumour tissues $(p<0.01$; figure 1B). Moreover, CA4 expression was silenced in all nine CRC cell lines tested (figure 1C). These results suggested an aberrant downregulation of CA4 in CRC. 
A

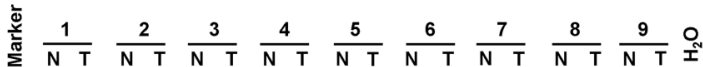

CA4

$\beta$-actin
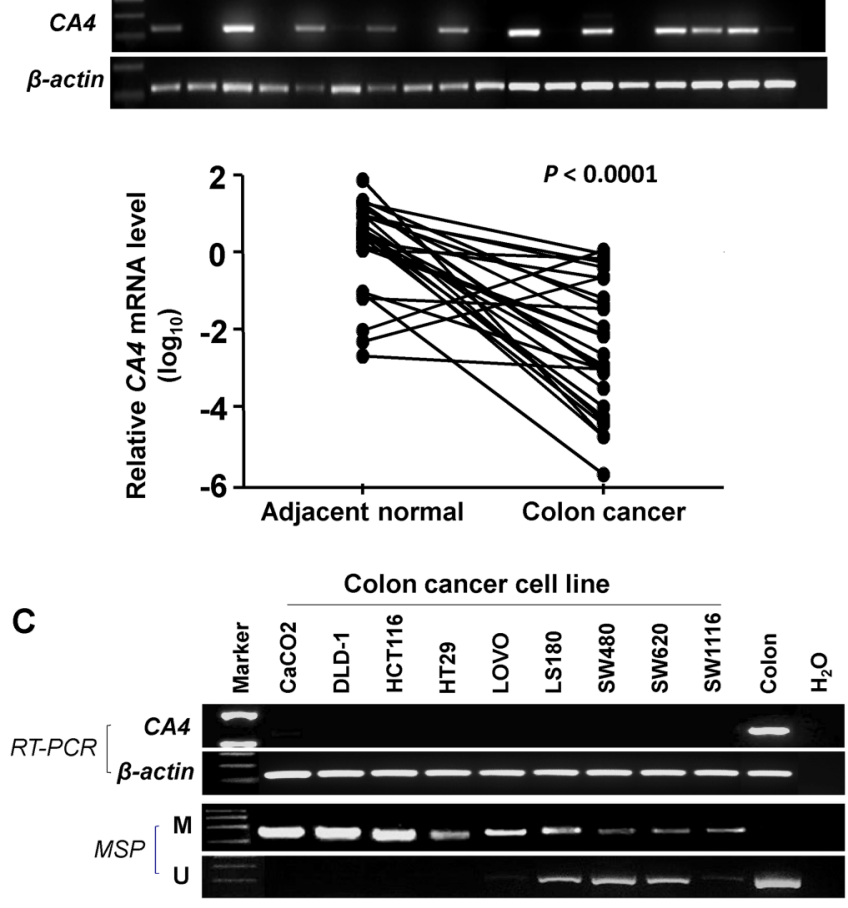

D

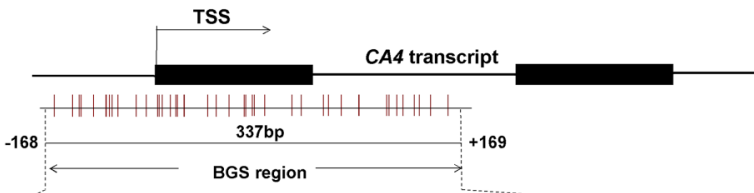

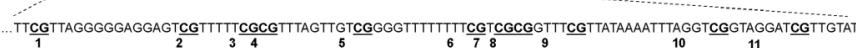

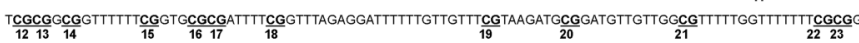

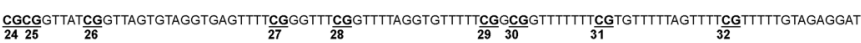
TTTTTCGCGGGCGATCGGTGAGCGTCGGTGGCGTTGGGGTTTCGGG

DLD-1 12345678910111213141516171819202122232425262728293031323334353637383940

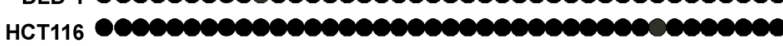

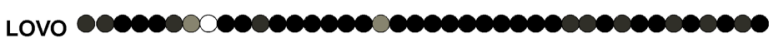

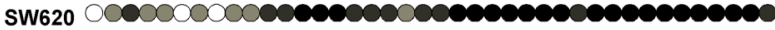
Colon 0000000000000000000000000000000000000000 ○ $0 \sim 24 \%$ - $25 \sim 49 \%$ - $50 \sim 74 \%$ - $75 \sim 100 \%$

E

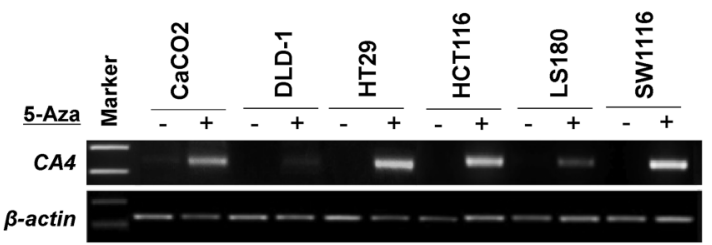

B
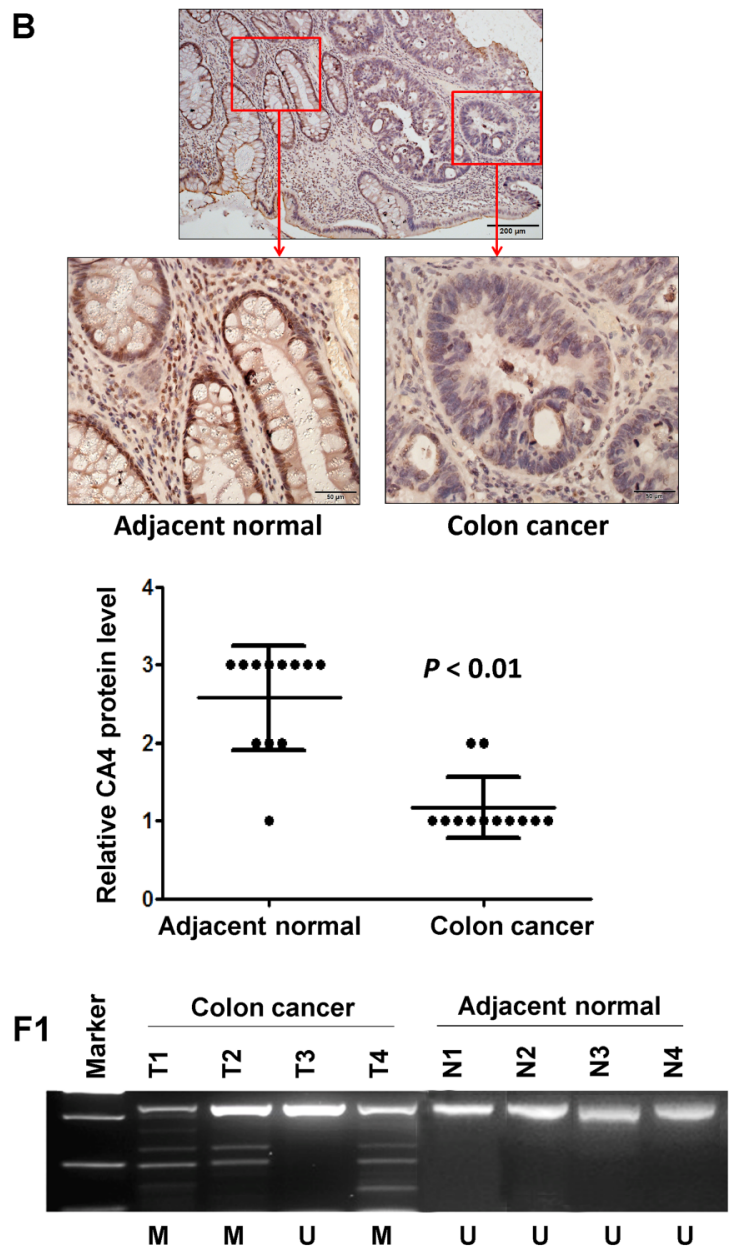

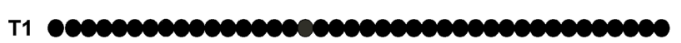

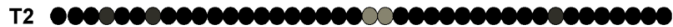
N1 0000000000000000000000000000000000000000 N2 0000000000000000000000000000000000000000

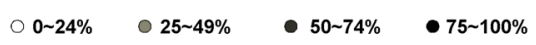

F2

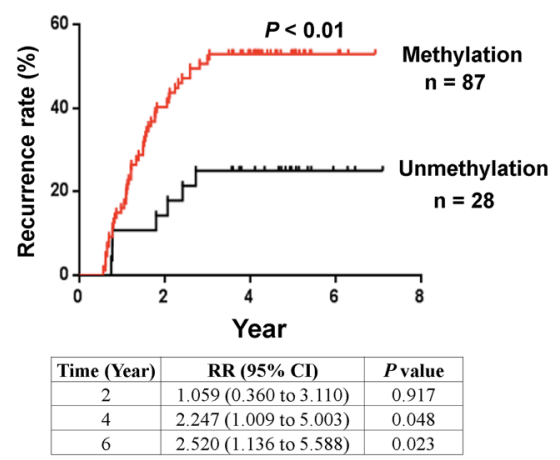

Figure 1 Promoter hypermethylation of CA4 led to the downregulation of CA4 in colorectal cancer (CRC) tissues and served as independent predictor of CRC recurrence. (A) Expression of CA4 mRNA was significantly downregulated in CRC tumour tissues compared with adjacent non-tumour tissues $(\mathrm{n}=27)$ (normalised to $\beta$-actin). (B) Representative images of immunohistochemical staining of CA4 protein expression in CRC tumour and adjacent normal tissues (upper panel). Quantification of CA4 protein expression by counting cells with positive nuclear staining $(n=12)$. (C) CA4 expression was lost or reduced in all colon cancer cell lines. Methylation-specific PCR was performed to determine the status of CA4 promoter methylation. ' $M$ ' indicates methylation while ' $U$ ' indicates unmethylation. (D) Bisulfite-sequencing was performed on the CA4 genomic region $(-168 \mathrm{bp}$ to $+169 \mathrm{bp})$ relative to transcription start site. Dense methylation was observed in CRC cell lines, but not in normal colon tissue. (E) The mRNA expression of CA4 was restored after treatment with demethylation agent 5-Aza-2'-deoxycytidine (5-Aza). (F1) Representative gel images of the CA4 methylation status in CRC tumour and adjacent non-tumour tissues determined by combined bisulfite restriction analysis (COBRA), and confirmed by bisulfite-sequencing. The digested fragments represent the methylated DNA (M), while the undigested fragment represents the unmethylated DNA (U). (F2) Patients with CA4 hypermethylation ( $n=87)$ had higher recurrence than those without methylation (U) $(n=28)$ based on the log-rank test $(p<0.01)$. 


\section{Transcriptional silence of $C A 4$ is mediated by promoter hypermethylation}

To determine the mechanism leading to the downregulation of CA4, we evaluated the methylation status of the CA4 promoter by methylation-specific PCR. Full or partial methylation was detected in all nine CRC cell lines but not in normal colon tissue (figure 1C). The detailed methylation status of the individual $\mathrm{CpG}$ sites within the $C A 4$ promoter region was then examined by bisulfite genomic sequencing (figure 1D). Consistent with methylation-specific PCR, dense methylation was confirmed in the CRC cell lines by bisulfite genomic sequencing (figure 1D). To further confirm that promoter methylation was involved in the silencing of CA4, six CRC cell lines (CaCO2, DLD-1, HT29, HCT116, LS180 and SW1116) with silenced CA4 expression were treated with the DNA methyltransferase inhibitor 5-Aza. A restored expression of CA4 was observed in all colon cancer cell cells detected (figure 1E). Our results therefore indicate that the transcriptional silence of CA4 was mediated by DNA methylation.

\section{CA4 is an independent predictor of the recurrence in patients with CRC}

To evaluate the association of CA4 methylation with the clinicopathological features and clinical outcomes of patients with CRC, we evaluated the $C A 4$ promoter hypermethylation status in 115 primary CRCs and in nine adjacent normal colon tissues by combined bisulfite restriction analysis (figure 1F1). Frequent hypermethylation was detected in $76 \%$ of the CRC tumour tissues (87/ 115 ) but not in any of the adjacent normal tissues (figure $1 \mathrm{~F} 1$ ). As shown in online supplementary table S2, there was no correlation between CA4 hypermethylation and age, gender, tumour localisation, differentiation and the TNM stage of the patients with CRC. In univariate Cox regression analysis, CA4 hypermethylation was associated with an increased risk of cancer recurrence (RR 2.63, 95\% CI 1.185 to $5.823 ; \mathrm{p}<0.05$ ) (table 1). After adjustment for potential confounding factors, multivariate Cox regression analysis showed that CA4 hypermethylation was an independent predictor of cancer recurrence in patients with CRC (RR 2.65, 95\% CI 1.193 to $5.902 ; \mathrm{p}<0.05$ ) (table 1). As shown in the recurrence curves, the patients with CRC with CA4 hypermethylation had a significantly higher risk of cancer recurrence $(\mathrm{p}<0.01$, log-rank test) (figure 1F2). Therefore, our results indicate that CA4 promoter hypermethylation is an independent predictor of CRC recurrence.

\section{CA4 suppresses CRC cell growth}

The frequent silencing of CA4 in CRC cell lines and tissues suggests that CA4 may have a tumour-suppressive function to promote CRC tumorigenesis. To prove this, we generated two stable transfected CRC cell lines (HCT116 and SW1116) with CA4 overexpression. Conversely, a normal human colon epithelial cell line (NCM460) with CA4 expression was used for loss of CA4 function by stable transfection of shCA4. The effects of CA4 ectopic expression and knockdown were verified by realtime (RT)-PCR and western blot, respectively (figure 2A). The ectopic expression of CA4 significantly suppressed cell viability in HCT116 and SW1116 compared with empty vectortransfected HCT116 and SW1116, while an inverse effect was observed in NCM460 with a knockdown of CA4 (figure 2B). In keeping with this, the number of colonies which had formed in CA4-transfected HCT116 and SW1116 were reduced significantly compared with empty vector-transfected HCT116 and SW1116, while knockdown CA4 promoted the colony formation ability in NCM460 (figure 2C). These results indicate that CA4 is important for inhibiting CRC cell growth.

To determine the mechanism by which CA4 inhibited cell growth, we analysed the effect of CA4 on cell cycle distribution by flow cytometry. The ectopic expression of CA4 in the CRC cells led to a significant increase in the G1 phase population, and a corresponding reduction in the $S$ phase population (figure 2D1). Consistently, CA4 reduced the protein expression of the G1-S transition promoter cyclin D1, but enhanced the G1 gatekeepers $\mathrm{p} 21^{\mathrm{Cip} 1}$ and $\mathrm{p} 27^{\mathrm{Kip} 1}$ (figure 2D2). These results suggest that CA4 acts on the G1/S checkpoint to prohibit the cell cycle in CRC cells.

\section{Ectopic expression of CA4 promotes cell apoptosis}

We further examined the contribution of apoptosis to the observed growth inhibition in CRC cells by CA4. Flow cytometry with annexin $\mathrm{V}$ and 7-AAD double staining was performed to analyse the effect of CA4 on apoptosis. The result showed an

Table 1 Cox regression analysis of potential recurrence predictor for patients with colon cancer

\begin{tabular}{|c|c|c|c|c|}
\hline \multirow[b]{2}{*}{ Variables } & \multicolumn{2}{|c|}{ Univariate Cox regression analysis } & \multicolumn{2}{|c|}{ Multivariate Cox regression analysis } \\
\hline & RR $(95 \%$ Cl) & $\mathrm{p}$ Value & RR $(95 \%$ Cl) & p Value \\
\hline Age & 1.007 (0.987 to 1.028$)$ & 0.481 & 1.005 (0.984 to 1.027$)$ & 0.629 \\
\hline \multicolumn{5}{|l|}{ Gender } \\
\hline M & 1.217 (0.689 to 2.149 ) & 0.498 & 1.290 (0.729 to 2.282$)$ & 0.382 \\
\hline $\mathrm{F}$ & 1.00 & & 1.00 & \\
\hline \multicolumn{5}{|l|}{ TNM } \\
\hline$\|$ & 1.00 & 0.545 & & \\
\hline III & 1.208 (0.656 to 2.225$)$ & & & \\
\hline \multicolumn{5}{|l|}{ Localisation } \\
\hline Colon & $1.078(0.628$ to 1.852$)$ & 0.785 & & \\
\hline Rectum & 1.00 & & & \\
\hline \multicolumn{5}{|l|}{ Differentiation } \\
\hline Low & 1.224 (0.664 to 2.255$)$ & 0.517 & & \\
\hline Moderate/high & 1.00 & & & \\
\hline \multicolumn{5}{|l|}{ CA4 methylation } \\
\hline Yes & 2.626 (1.185 to 5.823$)$ & 0.017 & 2.654 (1.193 to 5.902$)$ & 0.017 \\
\hline No & 1.00 & & 1.00 & \\
\hline
\end{tabular}




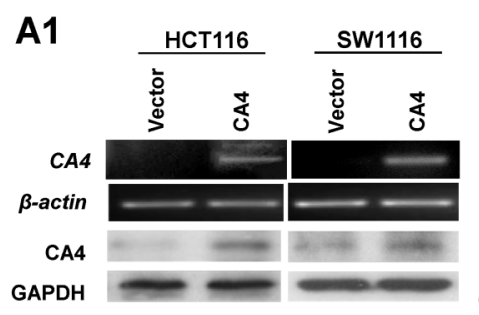

C1

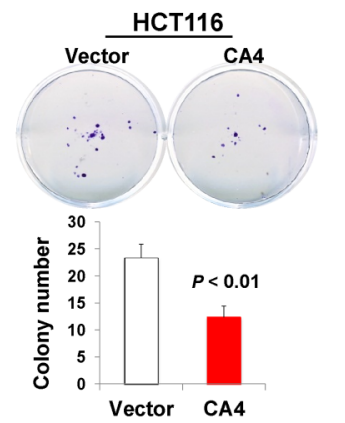

D1

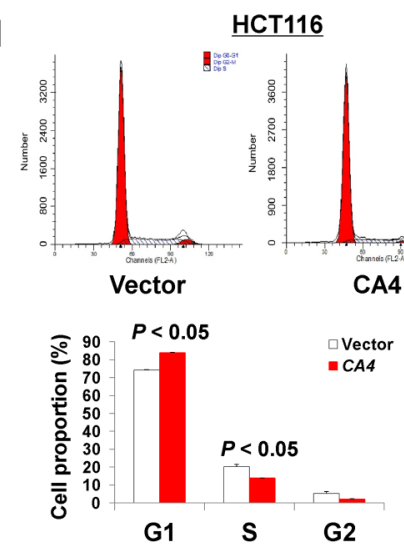

A2

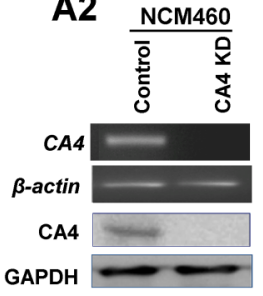

B1

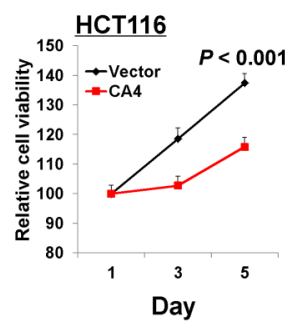

B2

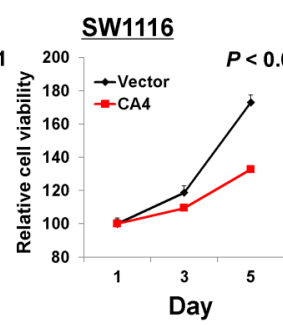

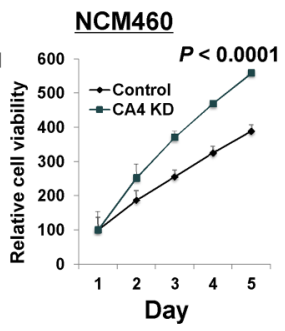

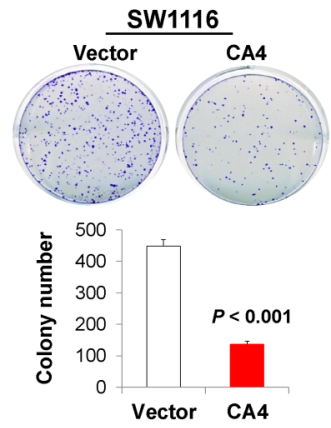

C2
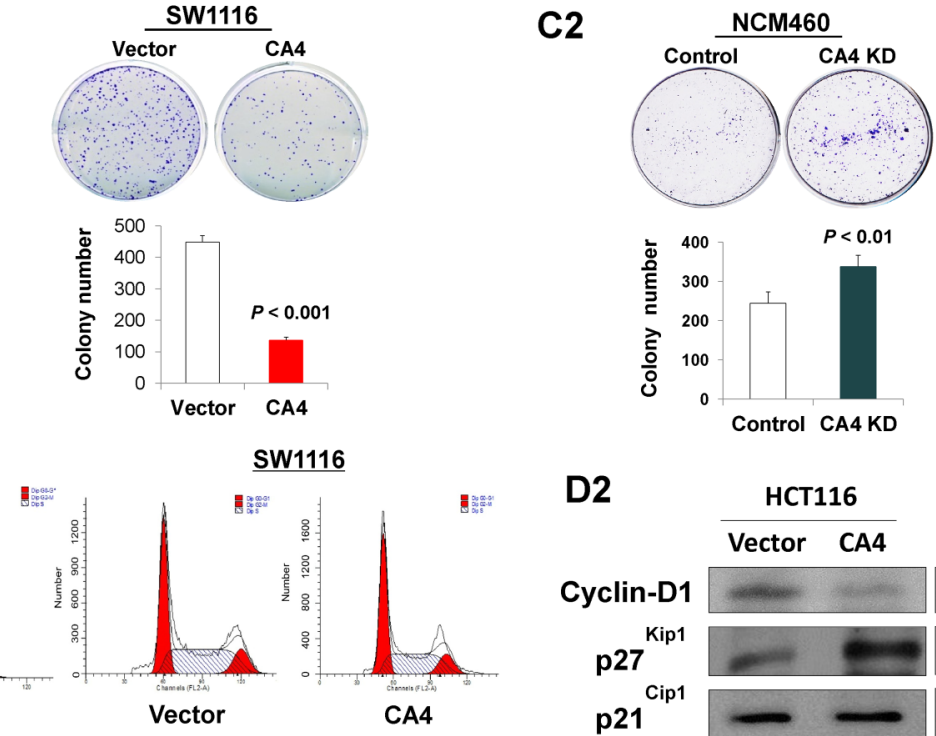

D2

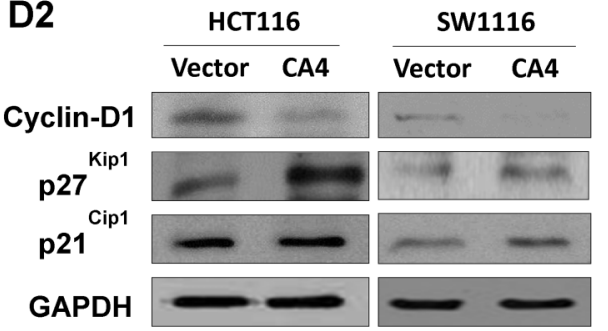

E2
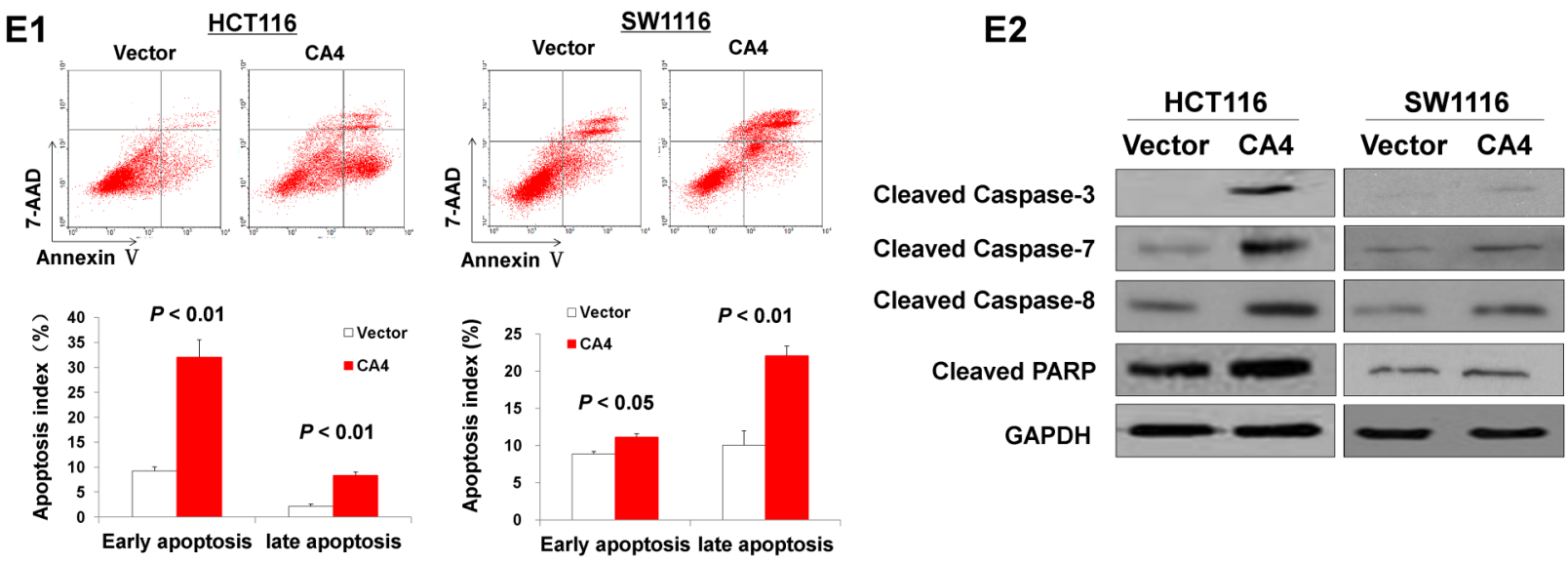

Figure 2 CA4 inhibited colorectal cancer cell growth and induced cell apoptosis. (A1) Ectopic expression of CA4 in HCT116 and SW1116 cell lines was confirmed by RT-PCR and western blot. (A2) Knockdown effect of CA4 by shCA4 in normal colon epithelial cell line NCM460 was confirmed by RT-PCR and western blot. (B1) Cell growth was inhibited by ectopic expression of CA4 in HCT116 and SW1116 cells. (B2) Knockdown of CA4 by shCA4 promoted the growth of NCM460 cells. (C1) Ectopic expression of CA4 suppressed colony formation in HCT116 and SW1116 cells. (C2) Knockdown of CA4 promoted colony formation in NCM460 cells. (D1) CA4 increased cell population at G1 phase but decreased at S phase by flow cytometry analysis in HCT116 and HCT1116 cells. (D2) Ectopic expression of CA4 reduced the protein level of cyclin-D1 while enhanced the level of p27 $7^{\mathrm{Kip} 1}$ and p21 ${ }^{\mathrm{Cip1}}$, but had no effect on p15 $5^{\mathrm{INK} 4 \mathrm{~B}}$ level. (E1) CA4 promoted cell apoptosis analysed by flow cytometry of cells stained with annexin V/7-Aminoactinomycin D (7-AAD). (E2) CA4 induced protein expression of the active forms of caspase-3, caspase-7 and caspase-8 by western blot.

increase in the number of early apoptotic cells $(32.01 \pm 3.50 \%$ vs $9.23 \pm 0.83 \%, \mathrm{p}<0.01)$ and the late apoptotic cells $(8.34 \pm 0.68 \%$ vs $2.15 \pm 0.47 \%, \mathrm{p}<0.01)$ in the CA4-transfected HCT116 cells compared with the vector-transfected HCT116 cells (figure 2E1). A similar effect was observed in the CA4-transfected SW1116 cells, which showed an increased 
proportion of cells in the early apoptotic phase $(11.32 \pm 0.46 \%$ vs $8.85 \pm 0.37 \%, \mathrm{p}<0.05)$ and in the late apoptotic phase $(22.08$ $\pm 1.30 \%$ vs $10.05 \pm 1.9 \%, \mathrm{p}<0.01)$ compared with the control cells (figure 2E1). We examined the key cell apoptosis regulators and found that CA4 significantly enhanced the protein levels of the active forms of caspase- 3 , caspase- 7 and caspase- 8 in the HCT116 cells and the SW1116 cells (figure 2E2). Therefore, our results indicate that CA4 favours the occurrence of apoptosis in CRC cells.

CA4 suppressed invasion and migration of colon cancer cells To investigate the effects of CA4 on colon cancer cell migration and invasion, the monolayer wound healing assay and Matrigel invasion assay were performed. The ectopic expression of CA4 markedly suppressed the cell migration ability in the HCT116 cells and the SW1116 cells (figure 3A). Quantitative analyses at $48 \mathrm{~h}$ confirmed a significant reduction in wound closure in the CA4-expressing cells compared with the control cells $(28 \%$ in the HCT116 cells and 53\% in the SW116 cells, respectively), thereby suggesting that the migration rate of the CA4-expressing cells was significantly lower than the control cells. Matrigel invasion assay also showed that CA4 significantly impaired the invasiveness of the HCT116 cells and the SW1116 cells (figure 3B). Moreover, the protein expression of the epithelial-mesenchymal transition (EMT) markers, including E-cadherin, $\mathrm{N}$-cadherin and Vimentin in the CA4-overexpressing cells, was examined by western blot. As shown in figure 3C, the ectopic expression of CA4 enhanced the protein levels of E-cadherin, while reducing $\mathrm{N}$-cadherin and Vimentin in the HCT116 cells and the SW1116 cells. Thus, CA4 suppresses the migration and the invasive abilities of colon cancer cells by modulating the key EMT regulating factors.

CA4 inhibits tumorigenicity in nude mice

To further explore the in vivo tumorigenic ability of CA4, empty vector-transfected and CA4-transfected HCT116 cells,
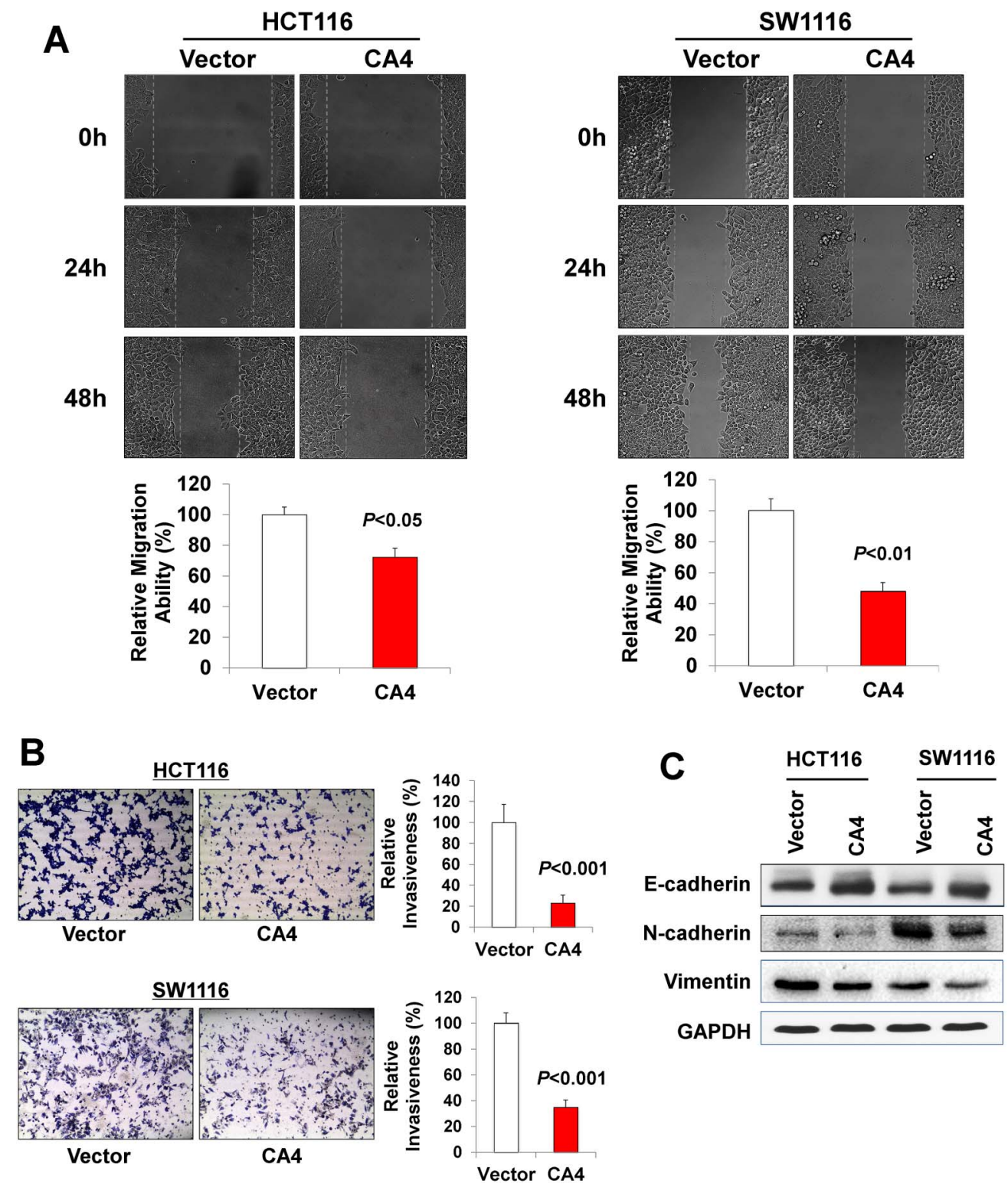

Figure 3 Expression of CA4 suppressed migration and invasion abilities of colorectal cancer (CRC) cells. (A) Expression of CA4 suppressed CRC cell migration. Wound-healing assay was performed and representative images are shown. (B) Expression of CA4 suppressed CRC cell invasion. Matrigel invasion assay was performed and representative images are shown. (C) The effect of CA4 expression on protein expression of key epithelialmesenchymal transition (EMT) markers by western blot. 
respectively, were injected into the right flanks of nude mice. The tumour growth rates in the nude mice injected with the HCT116-CA4 cells were significantly slower than in those injected with the HCT116-vector control cells (figure 4A). Eighteen days after injection, the mice were sacrificed and the xenografts were excised. The average tumour weight in the nude mice injected with HCT116-CA4 $(0.168 \pm 0.080 \mathrm{~g})$ was significantly lower compared with that in the control mice $(0.825 \pm 0.072 \mathrm{~g})(\mathrm{p}<0.01$, figure $4 \mathrm{~A})$. The CA4 expression in the CA4-transfected tumours was confirmed by RT-PCR and western blot, respectively (figure 4B), thus allowing the inference that CA4 suppressed the tumorigenesis in vivo.

Concomitant with the inhibition of cell proliferation in vitro, tumours from the mice injected with CA4-transfected cells displayed significantly reduced proliferative activity compared with the control littermates by Ki-67 immunostaining $(77.3 \pm 2.3 \%$ vs $54.4 \pm 1.5 \%, \mathrm{p}<0.005$; figure $4 \mathrm{C}$ ). Furthermore, TUNEL staining analysis showed that the apoptotic index significantly increased in tumour cells with CA4 overexpression compared with vector control $(28.6 \pm 5.5 \%$ vs $71.4 \pm 13.4 \%, \mathrm{p}<0.005$; figure $4 \mathrm{D})$. The results from the in vivo model provide further evidence of the tumour-suppressive role of CA4.

\section{CA4 directly interacts with WTAP and the tumour-suppressor} function of CA4 is WTAP-dependent

To further define the molecular basis mediated by CA4 to execute its tumour-suppressive function, we performed immunoprecipitation followed by mass spectrometry analysis to identify the interaction candidates of CA4 in CRC. WTAP was identified to be a CA4-interacting partner with functional interest (figure 5A). Reciprocal co-immunoprecipitation was performed to ascertain the functional interaction of CA4 and WTAP. As expected, a positive WTAP signal was observed in the immunoprecipitant pulled down by the CA4 antibody. Moreover, CA4 was also detected in the protein pool pulled down by the WTAP antibody (figure $5 \mathrm{~B}$ ). These results confirm the interaction between CA4 and WTAP. It was essential to evaluate whether WTAP would cooperate with CA4 to exert the inhibitory effect on cell proliferation. We examined the effect of WTAP knockdown on the CA4-mediated inhibition of cell proliferation. As shown in figure 5C, WTAP knockdown abolished the suppressive effect of CA4 on cell viability (figure 5C1) and on colony-formation abilities (figure $5 \mathrm{C} 2$ ). These results therefore indicate that CA4 exerts its tumour-suppressive function depending on the existence of WTAP.

\section{The tumour-suppressive function of CA4 is mediated} through the inhibition of the Wnt signalling pathway

Because the Wnt signalling pathway plays a significant oncogenic role in CRC, modulators on this pathway may alter the development and progression of CRC. Because CA4 interacted with WTAP in CRC, we hypothesised that CA4 might be involved in the regulation of the Wnt signalling pathway. To verify this, we performed luciferase reporter assay to determine the effect of CA4 on the activity of the Wnt signalling pathway. The ectopic expression of CA4 in CRC cell lines HCT116 and
A
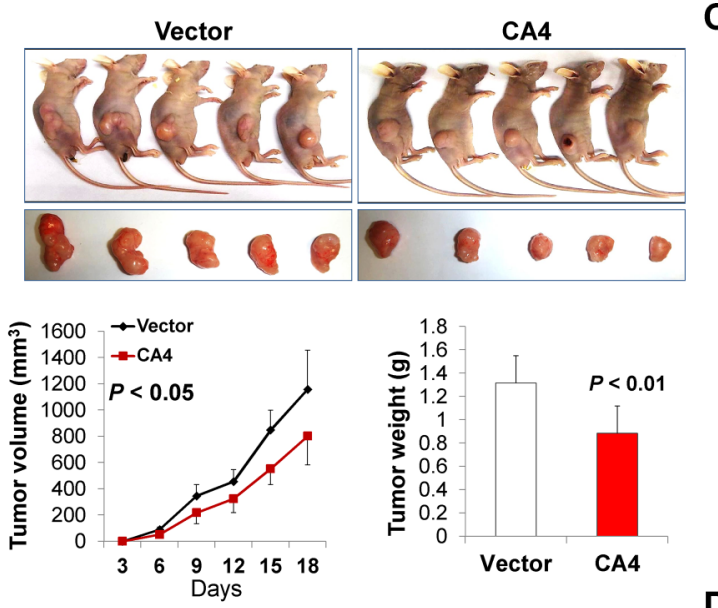

B
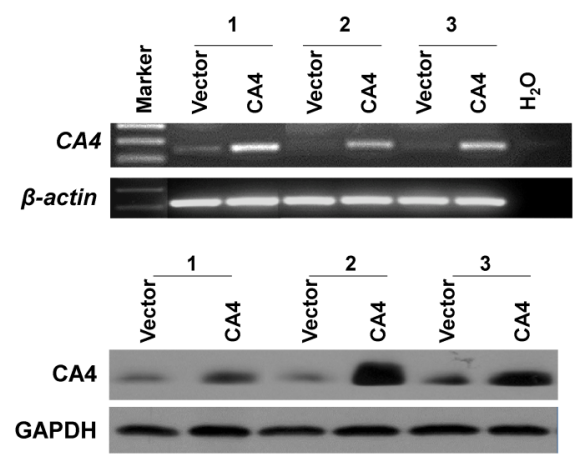

C
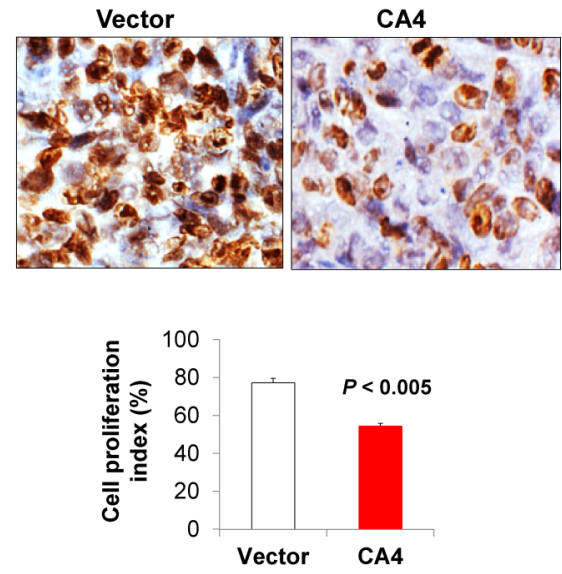

D
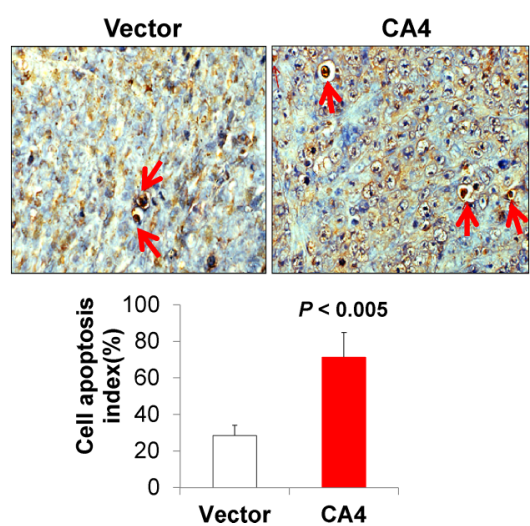

Figure 4 CA4 inhibited tumorigenesis in vivo. (A) Expression of CA4 reduced the tumour volume and tumour weight in nude mice. (B) CA4 expression in tumours from CA4-transfected nude mice was confirmed by RT-PCR and western blot. (C) Cell proliferation in tumour tissues isolated from CA4-expressing or control nude mice was determined by Ki-67 staining. Cell proliferation index was quantified by counting the proportion of Ki-67-positive cells. (D) Cell apoptosis in tumour tissues isolated from CA4-expressing or control nude mice was examined by TUNEL staining. Apoptosis index was quantified by counting the proportion of TUNEL-positive cells. 
A
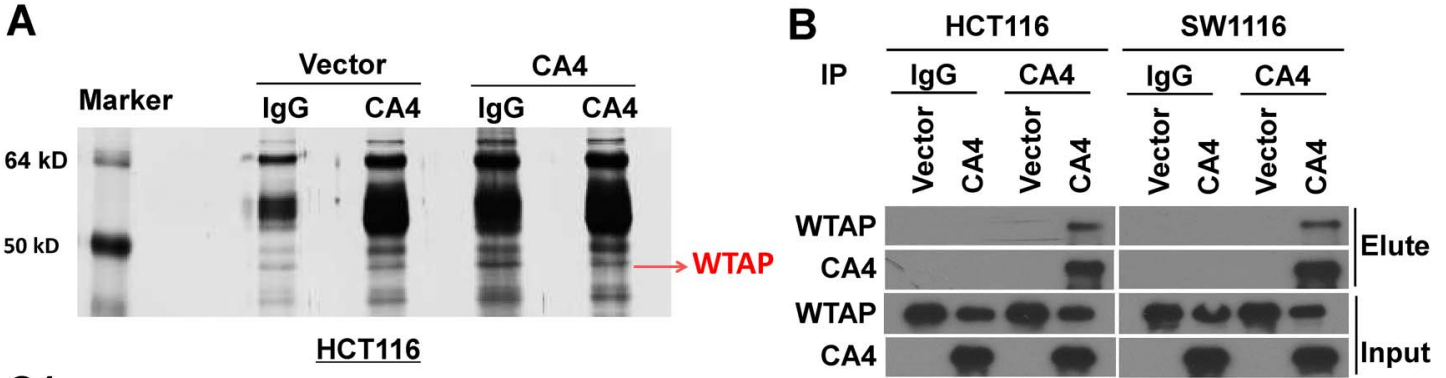

C1

HCT116

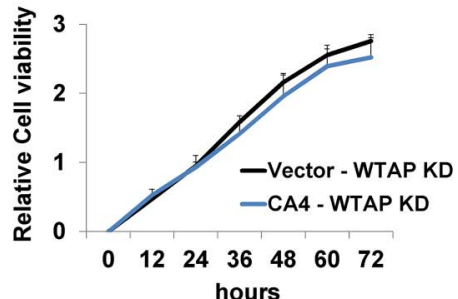

SW1116
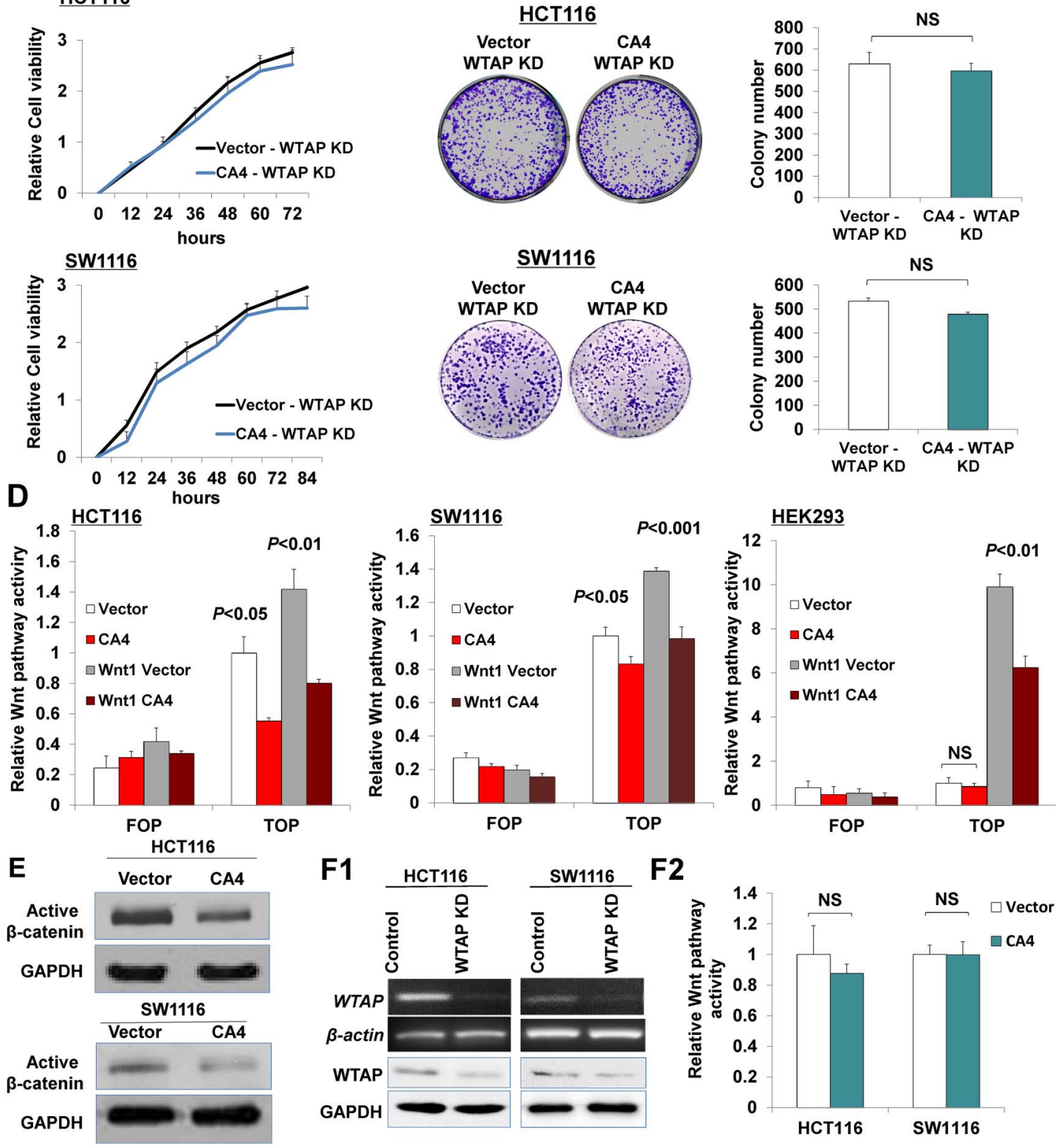

F1
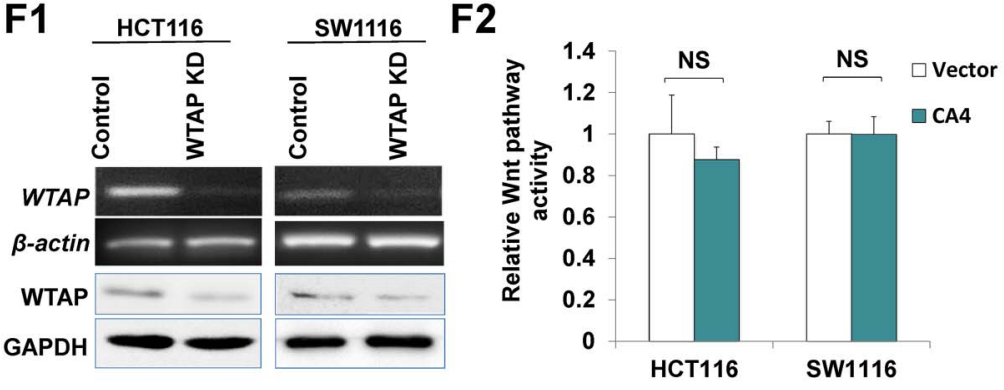

Figure 5 CA4 interacted with Wilms' tumour 1-associating protein (WTAP) and the repressed Wnt signalling pathway. (A) Immunoprecipitant of CA4 was analysed by sodium dodecyl sulfate-polyacrylamide gel electrophoresis (SDS-PAGE). Proteins were stained by silver staining. Proteins from region of interest were identified by mass spectrometry (indicated by arrow). (B) The interaction between CA4 and WTAP was confirmed by co-immunoprecipitation (IP). (C) Knockdown of WTAP abolished the growth-suppressive effect mediated by CA4 determined by cell viability assay (C1) and colony formation assay (C2) in HCT116 and SW1116 cells. (D) CA4 inhibited the Wnt signalling pathway in HCT116, SW1116 and HEK293 (control) cells with a pronounced effect after inducing Wnt signalling by Wnt1 transfection, as revealed by TOP-Flash luciferase reporter assays. HCT116, SW1116 and HEK293 cells were transiently transfected with pCDNA3.1 empty vector, pcDNA3.1-CA4, pCS2+/Wnt-1 plasmid, pCS2+/Wnt-1 + pcDNA3.1-CA4 and co-transfected with TOP-Flash plasmid or FOP-Flash plasmid for $6 \mathrm{~h}$. (E) Ectopic expression of CA4 reduced the level of active $\beta$-catenin by western blot. (F1) Knockdown effect of WTAP by siWTAP was evaluated by RT-PCR and western blot. (F2) Knockdown of WTAP abolished the suppressive effect of CA4 on the Wnt signalling pathway. 
SW1116 reduced the activity of the endogenous Wnt signalling pathway, as demonstrated by TOP-Flash luciferase reporter assays, but not in the normal HEK293 cells (figure 5D). Activating Wnt signalling is achieved by Wnt1 transfection in HCT116, SW1116 and HEK293, whereas the cotransfection of CA4 in these three cell lines dramatically inhibited the Wnt signalling pathway (figure 5D). In keeping with this, CA4 reduced the protein levels of active $\beta$-catenin in the HCT116 cells and the SW1116 cells, they being an essential component of the Wnt signalling pathway (figure 5E). WTAP knockdown by siWTAP abolished the suppressive effect of CA4 on the Wnt signalling pathway (figure $5 \mathrm{~F}$ ). These results suggest that CA4 inhibits the CRC growth through compromising the Wnt signalling pathway. The effect of CA4 on the inhibition of the Wnt signalling pathway is WTAP-dependent.

\section{CA4 induces WTAP protein degradation via polyubiquitination}

In the light of the interaction between CA4 and WTAP, we tested whether the ectopic expression of CA4 could alter the expression of WTAP. As shown in figure 6A1, WTAP mRNA levels were not changed in the CA4-transfected HCT116 cells and the SW1116 cells compared with the vector-transfected control cells. However, the WTAP protein levels were significantly reduced in both cell lines transfected with CA4 (figure 6A2). Moreover, CA4 reduced the WTAP protein levels of the cytoplasm and the nucleus (figure 6B), thereby implying that CA4 might be involved in mediating the degradation of WTAP. The ubiquitin proteasome system (UPS) and autophagy are two major mechanisms which mediate protein degradation. Because autophagy mediated the bulk degradation of proteins in membrane-bound vacuoles, and CA4 was not reported to be stored in membrane-bound organelles or vacuoles, autophagy was therefore less likely to mediate the degradation of WTAP. ${ }^{17}$ Instead, UPS should mediate the degradation of WTAP. To confirm this, we treated cells with MG132 which is an inhibitor of UPS. The protein level of WTAP was restored in the CA4-expressing HCT116 cells treated with MG132 (figure 6C). This finding suggests that UPS mediated the degradation of WTAP. To further confirm this, we examined ubiquitin on WTAP since polyubiquitination is a signature of protein being degraded via UPS. Consistently, we found that CA4 dramatically promoted the polyubiquitination of the endogenous WTAP proteins (figure 6D). Collectively, these results suggest that CA4 promotes WTAP degradation through stimulating polyubiquitination.

\section{CA4 inhibits the Wnt signalling pathway by activating WT1}

WTAP (WT1-associating protein), as its name implies, can interact with the WT1-suppressor protein. ${ }^{18} \mathrm{WT} 1$ is a negative regulator of the Wnt signalling pathway. ${ }^{19}$ In the light of our observation that the ectopic expression of CA4 promotes the degradation of WTAP, the degradation of WTAP caused by CA4 would release free WT1 which thus suppresses the Wnt signalling pathway. To confirm this hypothesis, co-immunoprecipitation was performed to determine the effect of CA4 on the interaction between WTAP and WT1. As expected, CA4 clearly reduced the level of the WTAP/WT1 protein complex, therefore releasing free WT1 (figure 6E). This was further confirmed by the DNA-binding activity of WT1 by using the electrophoretic mobility shift assay. As shown in figure 6F, CA4 enhanced the DNA-binding activity of WT1 towards the WT1-response element in nuclear extracts; while, when the CA4-expressing cells were treated with MG132, the enhancement of the DNA-binding activity of WT1 was abolished (figure 6F), thereby indicating that CA4 could release WT1 and increase the binding activity of WT1 to its nuclear elements.

\section{TBL1 is essential for the CA4-mediated degradation of $\beta$-catenin}

TBL1 is one of the downstream targets of $\mathrm{WT} 1^{13}$ and it regulates the degradation of $\beta$-catenin. ${ }^{14}$ We therefore speculated that CA4 could induce the expression of TBL1 in CRC cells. To verify this, ChIP-PCR was performed in stable CA4-expressing HCT116 cells and control HCT116 cells. We found that the ectopic expression of CA4 favoured WT1 binding to the promoter region of TBL1 (figure 6G1). CA4 also increased the TBL1 mRNA level, as determined by RT-PCR (figure 6G2). These data further confirmed the effect of CA4 in activating WT1 in CRC. Because TBL1 was reported to mediate the $\beta$-catenin level, we examined the effect of CA4 on $\beta$-catenin expression and the Wnt signalling activity under the condition of TBL1 knockdown by siTBL1 (see online supplementary figure S2A). As expected, the knockdown of TBL1 diminished the effect of CA4 on inhibiting the protein expression of the active form of $\beta$-catenin (figure $6 \mathrm{H} 1$ ). Apart from the protein level, luciferase reporter assay confirmed that the knockdown of TBL1 abolished the effect of CA4 on the inhibition of $\beta$-catenin activity (figure $6 \mathrm{H} 2$ ). The knockdown of TBL1 did not alter the WTAP expression (see online supplementary figure S2B). Collectively, our results demonstrate that TBL1 is essential for the CA4-mediated degradation of $\beta$-catenin.

\section{DISCUSSION}

In this study, we first demonstrated that CA4 was readily expressed in normal human colonic tissues, but frequently silenced in CRC cell lines and primary CRC tumour tissues. We showed that the silencing of CA4 was regulated by promoter hypermethylation. Demethylation treatment by DNA methyltransferase inhibitor 5-Aza successfully restored the expression of CA4, thereby indicating that the promoter hypermethylation of CA4 by DNA methyltransferase plays a key role in the transcriptional silence of CA4 in CRC.

A series of in vitro and in vivo functional experiments revealed that CA4 possesses a tumour-suppressive function in CRC. The overexpression of CA4 could suppress the cell proliferation in two CRC cell lines in vitro and in nude mice tumorigenesis in vivo; while the knockdown of CA4 promoted the cell proliferation. These results strongly suggest that CA4 acts as a tumour suppressor in CRC. This prompted us to address the underlying mechanism by which CA4 regulates cell growth. Cell cycle distribution analysis by fluorescence-activated cell sorting (FACS) revealed that the re-expression of CA4 arrested CRC cells in the G1 phase, with a concomitant reduction in cellular proliferation compared with the control cells. To explore the molecular mechanism underlying the G1 phase arrest, we studied the regulatory proteins which control the G1 checkpoint in the cell cycle. We found that G1 phase arrest by CA4 was associated with the downregulation of cyclin-D1, a G1 cyclin, and the upregulation of $\mathrm{p} 21^{\mathrm{Cip} 1}$ and $\mathrm{p} 27^{\mathrm{Kip} 1}$, two key Cdk inhibitors. ${ }^{20}$ Concomitantly with the inhibition of cell proliferation, the growth-inhibitory effect of CA4 was also related to the induction of apoptosis, as evidenced in the CA4-expressing cultured CRC cell lines and in xenografted tumours in the nude mice (figure 4). Apoptosis was mediated by the caspasedependent apoptosis pathway including casepase-8, casepase-3, casepase-7 and poly ADP ribose polymerase (PARP). Therefore, CA4 exerts its effect on regulating cell cycle and cell death to suppress tumour growth. The significance of CA4 on cell 
A1
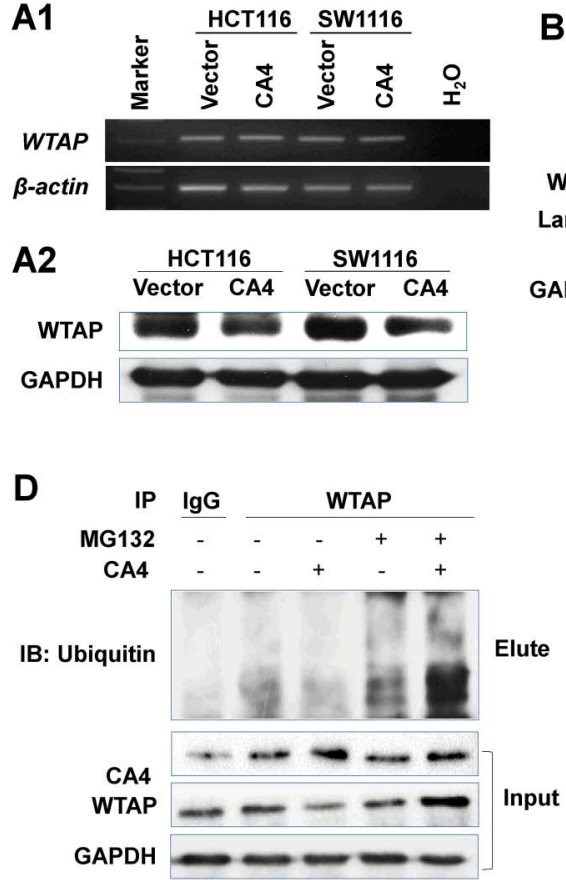

G1
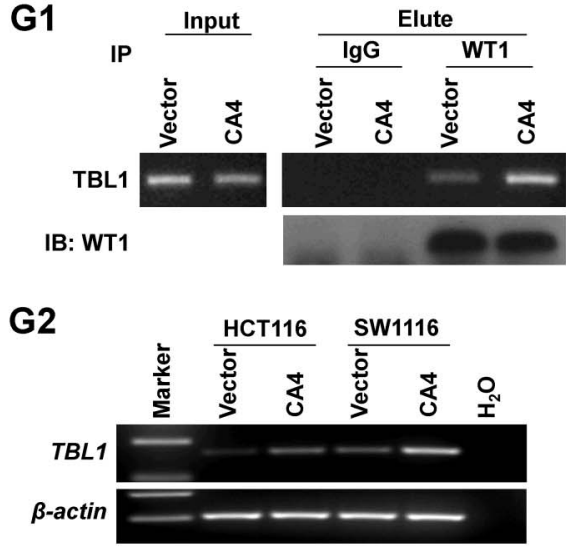

B

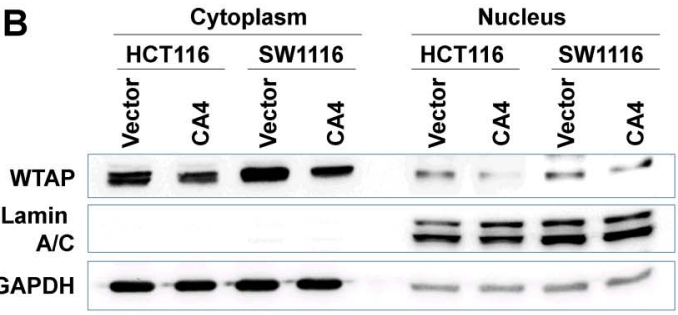

F

E

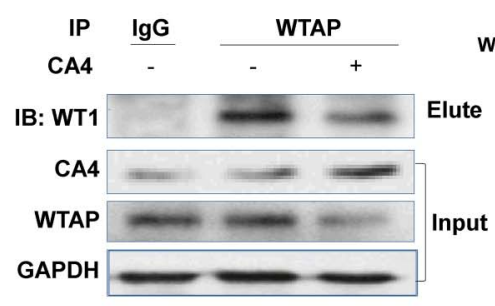

H1

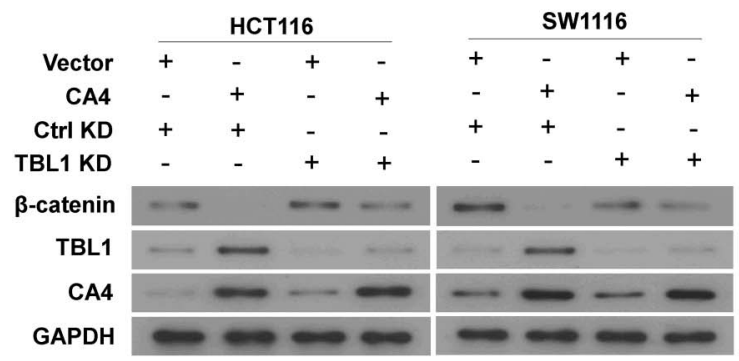

$\mathrm{H} 2$

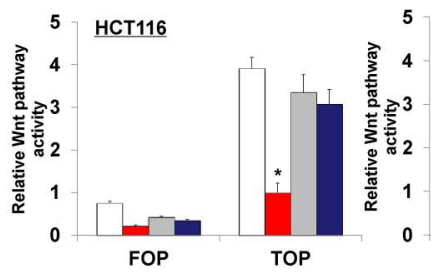

C

HCT116

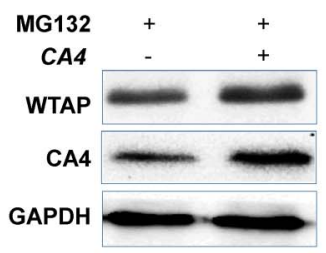

HCT116

IgG WT1 DMSO MG132 DMSO MG132

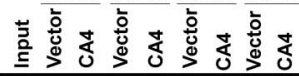

Figure 6 CA4 mediated the degradation of Wilms' tumour 1-associating protein (WTAP), resulting in the activation of WT1. (A1) Ectopic expression of CA4 did not change mRNA expression of WTAP. (A2) CA4 lowered protein expression of WTAP. (B) CA4 reduced nucleus and cytoplasm WTAP protein expression. Lamin A/C is a nuclear marker, while glyceraldehyde 3-phosphate dehydrogenase (GAPDH) is a cytoplasmic marker. (C) Treatment of MG132 $(10 \mu \mathrm{M})$ for $12 \mathrm{~h}$, an inhibitor of the ubiquitin proteasome system, abolished the effect of CA4 on the inhibition of WTAP protein expression. (D) Ectopic expression of CA4 promoted polyubiquitination of WTAP. Cell lysates were subjected to immunoprecipitation (IP) with anti-WTAP antibody or rabbit lgG as a control followed by immunoblotting with respective antibodies, as shown. Smear bands showed the polyubiquitination. (E) Ectopic expression of CA4 compromised the interaction between WTAP and WT1. (F) The interaction between WT1 and its DNA-binding element was revealed by electrophoretic mobility shift assay (EMSA). Ectopic expression of CA4 enhanced the level of WT1 associated with its DNA-binding elements. We isolated the WT1/DNA complex with CA4 antibody. The presence of the complex was analysed in a native polyacrylamide gel. The DNA was stained by SYBR green. The brightness of the band indicates the relative abundance of the WT1/DNA complex. This assay demonstrates the overall DNA-binding activity of WT1 with a significant difference between vector and CA4. (G1) Enrichment of WT1 in TBL1 promoter was found in CA4-expressing cells. Chromatin immunoprecipitation (ChIP)-PCR was performed to determine the interaction between WT1 and TBL1 promoter. (G2) Ectopic expression of CA4 enhanced the mRNA expression of TBL1. (H1) Knockdown of TBL1 by siTBL1 diminished the effect of CA4 on downregulation of $\beta$-catenin protein. (H2) HCT116 cells and SW116 cells were stable transfected with pCDNA3.1-CA4 or pcDNA3.1 empty vector. Knockdown of TBL1 by siTBL1 abolished the effect of CA4 on the inhibition of the Wnt signalling pathway determined by luciferase reporter assay. ${ }^{*} p<0.0001$.

migration and invasion was evaluated. The re-expression of CA4 in two colon cancer cell lines (HCT116 and SW1116) significantly inhibited their migration and invasion abilities. The molecular mechanisms by which CA4 exerts its anti-invasive function were shown to be mediated via enhancing the E-cadherin expression and inhibiting the $\mathrm{N}$-cadherin and Vimentin expression. E-cadherin functions as an invasion suppressor; while N-cadherin and Vimentin promote cell motility and invasion in cancers. ${ }^{21-23}$ Taken together, CA4 inhibits cell migration and invasion through modulating the key elements of EMT, such as E-cadherin, $\mathrm{N}$-cadherin and Vimentin, that contribute to its tumour-suppressive effect in CRC.

The overexpression of CA4 could reduce the level of cyclin-D1 which was regulated by $\beta$-catenin in the Wnt 
signalling pathway. This suggests that CA4 might be involved in the regulation of the Wnt signalling pathway. Indeed, luciferase reporter assay demonstrated that CA4 could suppress the Wnt signalling pathway in CRC cells. We thus investigated how CA4 governed the Wnt signalling pathway. We first identified WTAP as an interacting partner of CA4. WTAP was a negative regulator of WT1 which negatively regulated the Wnt signalling pathway. ${ }^{24}$ We identified that the tumour-suppressive effect of CA4 was dependent on WTAP; this was because the knockdown of WTAP abolished the suppressive effect of CA4 on cell proliferation in CRC cells. The ectopic expression of CA4 reduced the protein expression but did not alter the mRNA expression of WTAP. Moreover, CA4 led to the polyubiquitination of WTAP. Polyubiquitination is regarded as a tag for protein degradation. ${ }^{25}$ Proteins with polyubiquitination are subjected to degradation via proteasome. Together, these findings suggest that the interplay of CA4 and WTAP is important in regulating the stability of WTAP, which in turn activates WT1 to suppress the Wnt signalling pathway (figure 7).

WT1 is a transcription factor that governs the expression of a set of effectors. ${ }^{26}$ A genome-wide screening analysis identified that WT1 also governs the expression of the genes which regulate the Wnt signalling pathway. ${ }^{13}$ TBL1 is one of the downstream targets controlled by WT1. We demonstrated that the overexpression of CA4 promotes the interaction between WT1 and promoter of TBL1 by ChIP-PCR and increased the expression of TBL1. Moreover, the knockdown of TBL1 could abolish the effect of CA4 on inhibiting $\beta$-catenin activity (figure $6 \mathrm{H}$ ). TBL1 has been reported to regulate the degradation of $\beta$-catenin, ${ }^{27}$ thus TBL1 is essential for the CA4-mediated degradation of $\beta$-catenin. TBL1 is important for mediating the degradation of $\beta$-catenin in CRC.

Collectively, our findings demonstrate that CA4 promotes WTAP degradation by stimulating polyubiquitination, which in turn activates WT1 and therefore promotes the interaction between WT1 and its downstream transcriptional effector TBL1. The enhanced expression of TBL1 by WT1 therefore leads to the degradation of $\beta$-catenin. The reduced level of $\beta$-catenin results in the decreased activity of the Wnt signalling

pathway. All of these provide a mechanistic feature for explaining the involvement of CA4 in suppressing the Wnt signalling pathway (figure 7).

Other mechanisms of action of WT1 have been reported. During developmental and homoeostatic events, the Wnt signalling mechanism can be regulated by Dishevelled (Dvl1) which inhibits GSK3 $\beta$ activity and reduces the degree of $\beta$-catenin degradation. ${ }^{28}$ Therefore, a modulator, such as the WT1-induced inhibitor of Dvl1 (WID), can inhibit the activity of Dvl1 and regulate the activity of the Wnt signalling mechanism. ${ }^{19}$ Moreover, ebi (also called TBL1) induced by p53 can mediate the degradation of $\beta$-catenin regardless of its phosphorylation status. ${ }^{14}$ Therefore, the regulation of the Wnt signalling mechanism is complex. Our study shows that there is an additional regulation mechanism of WT1 involved in regulating Wnt signalling.

To ascertain the clinical application of CA4 in colon tumorigenesis in vivo, we examined the promoter methylation of CA4 in 115 patients with CRC. CA4 hypermethylation was detected in $76 \%$ of these CRC cases. Thus, CA4 promoter hypermethylation is a common event in colorectal carcinogenesis. The clinical outcomes of CRC vary greatly, depending on the growth status and aggressiveness of the individual tumour. One of the main outcomes is the recurrence of the disease. At present, many patients succumb to disease recurrence, especially those with Stage II or Stage III CRCs. These patients belong to a high-risk group for tumour recurrence. In one report, local recurrence occurred within 2 years and 3 years in $59.9 \%$ and $82.4 \%$ of the patients, respectively. ${ }^{29}$ Apart from carcinoembryonic antigen (CEA), few non-invasive markers are available for the monitoring or prognostication of patients with CRC. It is therefore crucial to identify predictive biomarkers that can provide better risk assessment for CRC recurrence. The Wnt signalling pathway associates with the capacity of colon cancer recurrence. ${ }^{30}$ We found that CA4 regulated the stability of $\beta$-catenin which was a central hub of the Wnt signalling pathway. We examined the influence of CA4 hypermethylation on the outcomes of patients with CRC and revealed that CA4 hypermethylation was associated with tumour relapse. Multivariate Cox
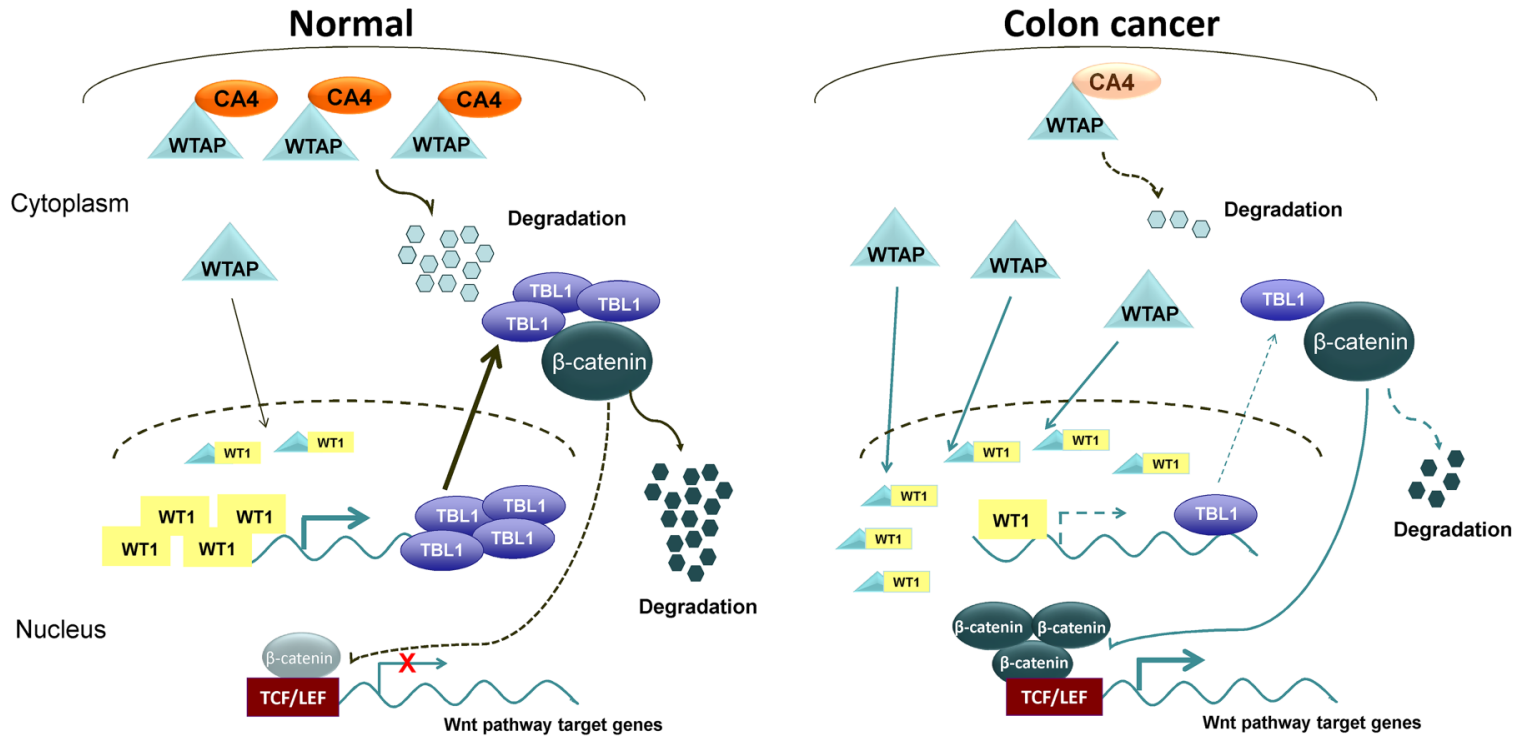

Figure 7 Proposed mechanistic scheme of CA4 suppressing the Wnt signalling pathway in colorectal cancer. CA4 promoted Wilms' tumour 1 -associating protein (WTAP) degradation by stimulating polyubiquitination, which in turn releases WT1 and therefore promotes the interaction between WT1 and TBL1 promoter. Induction of TBL1 mediates the degradation of $\beta$-catenin, and therefore suppresses the Wnt signalling pathway. 
regression analysis indicated that CA4 hypermethylation could represent as an independent predictor for the recurrence of patients with Stage II/III CRC. Therefore, these findings suggest that CA4 has clinical implications as a biomarker for disease recurrence.

In conclusion, our study uncovered for the first time the tumour-suppressive role of CA4 in CRC, and the mechanisms involved in cell cycle arrest and apoptosis induction. We also provide further evidence that CA4 suppresses the Wnt/ $\beta$-catenin signalling pathway by a direct interplay with WTAP and by targeting the WTAP-WT1-TBL1 axis. The detection of CA4 hypermethylation may serve as an independent biomarker for the recurrence of CRC.

Contributors IZ performed the experiments, analysed the data and drafted the manuscript. HT performed the experiments and also drafted the manuscript. $X L, H W$, JG, KW and MYYG performed the experiments. SCN and FKLC collected the samples and revised the manuscript. JJYS reviewed the data and commented on the manuscript. JY designed and supervised the studies and also wrote the paper.

Funding This project was supported by 863 Program China (2012AA02A506), 973 Program China (2013CB531401), the Shenzhen Technology and Innovation Project Fund, Shenzhen (JSGG20130412171021059), the Natural Science Foundation of China (NSFC) $(81201963,81372600)$, the Shenzhen Municipal Science and Technology R \& D Fund (JCYJ20120619152326450) and the Shenzhen Virtual University Park Support Scheme to CUHK Shenzhen Research Institute.

Competing interests None declared.

Patient consent Obtained.

Ethics approval Human Ethics Committee of Beijing University Cancer Hospital. Provenance and peer review Not commissioned; externally peer reviewed.

Open Access This is an Open Access article distributed in accordance with the Creative Commons Attribution Non Commercial (CC BY-NC 4.0) license, which permits others to distribute, remix, adapt, build upon this work non-commercially, and license their derivative works on different terms, provided the original work is properly cited and the use is non-commercial. See: http://creativecommons.org/ licenses/by-nc/4.0/

\section{REFERENCES}

1 Grady WM, Carethers JM. Genomic and epigenetic instability in colorectal cancer pathogenesis. Gastroenterology 2008;135:1079-99.

2 Cunningham D, Atkin W, Lenz HJ, et al. Colorectal cancer. Lancet 2010;375:1030-47

3 O'Connell MJ, Campbell ME, Goldberg RM, et al. Survival following recurrence in stage II and III colon cancer: findings from the ACCENT data set. J Clin Oncol 2008:26:2336-41.

4 Brock MV, Hooker CM, Ota-Machida E, et al. DNA methylation markers and early recurrence in stage I lung cancer. N Engl J Med 2008;358:1118-28.

5 Ahn JB, Chung WB, Maeda 0 , et al. DNA methylation predicts recurrence from resected stage III proximal colon cancer. Cancer 2011;117:1847-54.

6 Sapari NS, Loh M, Vaithilingam A, et al. Clinical potential of DNA methylation in gastric cancer: a meta-analysis. PLOS ONE 2012;7:e36275.
7 Okazaki S, Ishikawa T, Ilda $\mathrm{S}$, et al. Clinical significance of UNC5B expression in colorectal cancer. Int J Oncol 2012;40:209-16.

8 Okuyama T, Batanian JR, Sly WS. Genomic Organization and Localization of Gene for Human Carbonic Anhydrase-Iv to Chromosome-17q. Genomics 1993;16:678-84.

9 Tashian RE. The carbonic anhydrases - widening perspectives on their evolution, expression and function. Bioessays 1989;10:186-92.

10 Fleming RE, Parkkila S, Parkkila AK, et al. Carbonic anhydrase IV expression in rat and human gastrointestinal tract regional, cellular, and subcellular localization. J Clin Invest 1995:96:2907-13.

11 Purkerson JM, Schwartz GJ. Expression of membrane-associated carbonic anhydrase isoforms IV, IX, XII, and XIV in the rabbit: induction of CA IV and IX during maturation. Am J Physiol Regul Integr Comp Physiol 2005;288:R1256-63.

12 Wang N, Chen Y, Han YC, et al. Proteomic analysis shows down-regulations of cytoplasmic carbonic anhydrases, CAI and CAll, are early events of colorectal carcinogenesis but are not correlated with lymph node metastasis. Tumori 2012;98:783-91.

13 Kim MKH, McGarry TJ, Broin PO, et al. An integrated genome screen identifies the Wnt signaling pathway as a major target of WT1. Proc Natl Acad Sci USA 2009;106:11154-9.

14 Matsuzawa S, Reed JC. Siah-1, SIP, and Ebi collaborate in a novel pathway for beta-catenin degradation linked to p53 responses. Mol Cell 2001;7:915-26.

15 Lee TI, Johnstone SE, Young RA. Chromatin immunoprecipitation and microarray-based analysis of protein location. Nat Protoc 2006;1:729-48.

16 Drummond IA, Rupprecht HD, Rohwernutter $P$, et al. DNA Recognition by Splicing Variants of the Wilms-Tumor Suppressor, Wt1. Mol Cell Biol 1994;14:3800-9.

17 Dengiel J, Kristensen AR, Andersen JS. Ordered bulk degradation via autophagy. Autophagy 2008;4:1057-9.

18 Small TW, Bolender Z, Bueno C, et al. Wilms' tumor 1-associating protein regulates the proliferation of vascular smooth muscle cells. Circ Res 2006;99:1338-46.

19 Kim MS, Yoon SK, Bollig F, et al. A Novel Wilms Tumor 1 (WT1) Target Gene Negatively Regulates the WNT Signaling Pathway. J Biol Chem 2010;285:14585-93.

20 Besson A, Dowdy SF, Roberts JM. CDK inhibitors: Cell cycle regulators and beyond. Dev Cell 2008;14:159-69.

21 Canel M, Serrels A, Frame MC, et al. E-cadherin-integrin crosstalk in cancer invasion and metastasis. J Cell Sci 2013;126:393-401.

22 Breier G, Grosser M, Rezaei M. Endothelial cadherins in cancer. Cell Tissue Res 2014;355:523-7

23 Pan TL, Wang PW, Huang CC, et al. Network analysis and proteomic identification of vimentin as a key regulator associated with invasion and metastasis in human hepatocellular carcinoma cells. J Proteomics 2012;75:4676-92.

24 Little NA, Hastie ND, Davies RC. Identification of WTAP, a novel Wilms' tumour 1-associating protein. Hum Mol Gen 2000;9:2231-9.

25 Glickman $\mathrm{MH}$, Ciechanover $\mathrm{A}$. The ubiquitin-proteasome proteolytic pathway: Destruction for the sake of construction. Physiol Rev 2002;82:373-428.

26 Hohenstein P, Hastie ND. The many facets of the Wilms' tumour gene, WT1. Hum Mol Gen 2006;15:R196-201.

27 Gao CX, Xiao GT, Hu J. Regulation of Wnt/beta-catenin signaling by posttranslational modifications. Cell Biosci 2014;4:13.

28 Gao C, Chen YG. Dishevelled: The hub of Wnt signaling. Cellular Signalling 2010;22:717-27.

29 Yun HR, Lee LJ, Park JH, et al. Local recurrence after curative resection in patients with colon and rectal cancers. Int I Colorectal Dis 2008:23:1081-7.

30 Paez D, Gerger A, Zhang W, et al. Association of common gene variants in the WNT/beta-catenin pathway with colon cancer recurrence. Pharmacogenomics J $2014 ; 14: 142-50$ 